\title{
Reducing Uncertain Systems and Behaviors
}

\author{
Carolyn Beck * John Doyle ${ }^{\dagger}$ \\ EXTENDED ABSTRACT
}

\section{Introduction}

This paper considers the problem of reducing the dimension of a model for an uncertain system whilst bounding the resulting error. Model reduction methods with guaranteed upper error bounds have previously been established for uncertain systems described by a state-space type realization ([1], [2], [3], [4], [5], [6]); specifically, by a Linear Fractional Transformation (LFT) of a constant realization matrix over a structured uncertainty operator. In contrast to traditional 1-D model reduction where upper bounds on reduction are matched with comparable lower bounds, in the uncertain system problem there have previously been no lower bounds established. The computation of both upper and lower bounds is discussed in this paper, including a discussion of the use of Hankel-like matrices. These model reduction methods and error bound computations are then discussed in the context of kernel representations of behavioral uncertain systems. Due to space constraints only an extended abstract is given here; to obtain the full paper see http://avalon.caltech.edu/cds/cgibin/reports.cgi, or contact the first author.

Department of Electrical Engineering, University of Pittsburgh, 348 Benedum Hall, Pittsburgh, PA 15261 beck@ee.pitt.edu

Control and Dynamical Systems, M/S 116-81, California Institute of Technology, Pasadena, CA 91125

\section{Summary of the Paper}

We begin the paper with a brief review of the LFT modelling framework commonly used to represent uncertain systems, followed by a short discussion of existing model reduction results for uncertain systems. In particular, we consider the LFT paradigm shown in Figure 1 , where $\Delta$ represents uncertainty, or a dynamic element, and

$$
M=\left[\begin{array}{ll}
A & B \\
C & D
\end{array}\right]
$$

is a realization of the input-output mapping

$$
\Delta \star M=D+C \Delta(I-A \Delta)^{-1} B
$$

we assume throughout that the inverse is welldefined. If we let $\Delta$ represent repeated copies of the integral or shift operator (e.g., $1 / s$ ) then we recover the transfer function $(1 / s) \star M=$ $D+C(s I-A)^{-1} B$ and a standard state-space realization with state $x$, input $u$ and output $y$. By simply allowing the $\Delta$ block to represent more general system operators, LFT systems provide a convenient framework for adding uncertainty in which essentially all of the major state space results can then be generalized (see [7] and the references therein).

We assume $\Delta$ lies in a prescribed set,

$$
\Delta=\left\{\operatorname{diag}\left[\delta_{1} I_{n_{1}}, \ldots, \delta_{p} I_{n_{p}}\right]: \delta_{i} \in \mathcal{L}\left(l_{2}\right)\right\} .
$$

We often consider $\Delta$ which lie in a unity normbounded subset of $\boldsymbol{\Delta}$, denoted by $\mathbf{B}_{\Delta}$. Note that $\delta_{i} \in \mathcal{L}\left(l_{2}\right)$ allows time-varying operators 


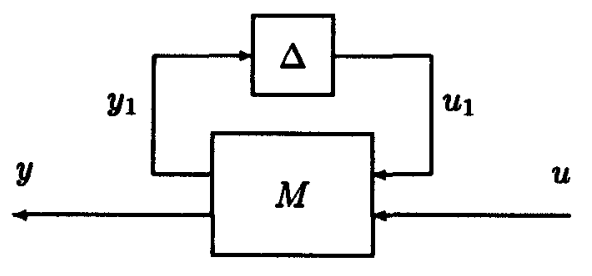

Figure 1: Uncertain System

on $l_{2}$, which are not commutative. Furthermore, it is typically assumed that $M$ is an LTI system, but it is equivalent and simpler to assume that $M$ is a constant and include the shift or integral operator as one of the $\delta_{i}$. This then gives us a state-space realization for uncertain systems which is analogous to standard or 1-D realizations.

In [2] and [3] it is shown that a general version of similarity transformations, system Lyapunov equations, and controllability and observability Gramians in balanced truncation model reduction and in terms of quantifying system minimality hold for uncertain systems modelled using the LFT framework. Namely, given a realization $(\Delta, M)$ and any $\epsilon \geq 0$, a lower order realization $\left(\Delta_{r}, M_{r}\right)$ exists such that $\sup _{\Delta \in \mathbf{B}_{\Delta}} \|(\Delta \star M)-\left(\Delta_{r} \star M_{r} \|_{l_{2} \rightarrow l_{2}} \leq \epsilon\right.$ if and only if there exist block diagonal structured solutions, $X \geq 0$ and $Y \geq 0$, to the system Lyapunov inequalities:

$$
\begin{aligned}
A Y A^{*}-Y+B B^{*} & \leq 0 \\
A^{*} X A-X+C^{*} C & \leq 0,
\end{aligned}
$$

where $\lambda_{\min }(X Y)=\epsilon^{2}$ with multiplicity corresponding to the difference in the dimensions of the full and reduced realizations. Existing LMI solvers may thus be used to find feasible solutions to (2). In the case where $\epsilon=0$, we can obtain a minimality result, and also a decomposition structure (see [3] for details). A more thorough summary of these and related realization theory results is presented in the full paper.

Computational methods are presented for reducing uncertain system models with the guaranteed upper error bounds mentioned above. Ideally, we would like to find minimum rank, structured Gramians $Y \geq 0$ and $X \geq 0$ to the above LMIs, i.e., solutions $Y$ and $X$ for which the product $Y X$ has the smallestvalued minimum eigenvalue with the highest multipicity. Although feasible solutions, $X$ and $Y$, are easily computed using convex programming methods (or any of the recent LMI solvers $[8,9]$ ), the optimization problem itself is a reduced rank LMI problem and as such does not yield a convex optimization problem, thus we cannot directly apply LMI algorithms to obtain solutions. However, we have constructed a straightforward heuristic algorithm using existing LMI techniques to obtain solutions for the model reduction and minimality problem, which have given quite good results not only in numerical tests, but in applications as well. A detailed description of the algorithm and an analysis of the test results are given in the full paper.

Although we can compute guaranteed upper error bounds using these methods, we cannot simultaneously compute lower bounds. For standard 1-D continuous systems, both upper and lower error bounds for balanced truncation model reduction can be computed using the singular values of the associated Hankel operator. In the case of uncertain and discrete time systems, the actual system Gramians are not used, but instead nonunique solutions to the LMIs in (2) are found. Thus we cannot strictly relate the solutions $Y$ and $X$ for the LMIs to a system Hankel operator and Hankel singular values. However, we may construct Hankel matrices for the uncertain systems we consider using the realization matrix $M$.

In the full paper, we discuss the relevance of Hankel matrices for uncertain systems, mainly in the context of minimality, followed by a discussion of associated Hankel operators and the use of such in computing lower bounds on system norms and for reduction. We define Hankel matrices for uncertain systems in a manner similar to those defined for formal power series [10]; structured Hankel matrices are also 
considered. We use the so-called controllability and observability matrices for uncertain systems defined in [3] for the construction of these Hankel matrices for uncertain systems. The singular values of the Hankel operators we construct provide reasonable lower bounds for the system norm, but appear to be conservative for model reduction lower bounds. Alternate approaches to computing model reduction lower bounds are also discussed in the full paper.

From the reduction and realization theory developed for uncertain input-output models up to this point, we then discuss computing reduced models with error bounds and minimality for kernel representations of behavioral uncertain systems. We consider the behavioral framework originally proposed by Willems [11]. In order to incorporate uncertainty into our models, we adopt the output nulling or kernel representation defined by Weiland [12] to describe 1-D behavioral systems. In this framework, both minimality and the evaluation of model reduction error bounds become more complex. For example, in the input-output framework, we have necessary and sufficient LMI conditions for minimality and model reduction bounds. In the behavioral framework, the LMI conditions are only sufficient. In fact, even in the 1-D behavioral case (i.e., no uncertainty) there exist only sufficient conditions. Additionally, if we consider a kernel representation of a behavior, then minimality also involves the issues of output injection and detectability, and if we consider model reduction of a kernel representation, then the error bounds should be interpreted in a gap-like metric. Furthermore, to apply the model reduction techniques previously described, stability and contractiveness of the uncertain behavioral representations, $(\Delta, M)$, are desired. Stable $M$ generalizes the use of stable coprime factor representations for input-output systems and as such norms can be used to define generalizations of normalized coprime factors.

In the full paper we address these issues, first introduced in [13], that are associated with normalization and minimality for uncertain behavioral system representations in more detail. Algorithms and associated upper error bounds for model reduction of behavioral uncertain systems are also discussed.

\section{Acknowledgements}

The first author would like to thank Lennart Andersson for sharing in numerous discussions related to the topics in this paper.

\section{References}

[1] W. Wang, J. Doyle, C. Beck, and K. Glover, "Model reduction of LFT systems," in Proc. IEEE Control and Decision Conference, 1991.

[2] C. L. Beck, J. Doyle, and K. Glover, "Model reduction of multi-dimensional and uncertain systems," IEEE Transactions on Automatic Control, 1995. (accepted).

[3] C. Beck, Model Reduction and Minimality for Uncertain Systems. PhD thesis, California Institute of Technology, 1995.

[4] A. Helmersson, Methods for robust gain scheduling. PhD thesis, Linkoping University, 1995.

[5] X. Chen and J. T. Wen, "Model reduction of multidimensional positive real systems," in Proc. IEEE Control and Decision Conference, 1994.

[6] L. Andersson and C. Beck, "Model comparison and simplification," in Proc. IEEE Control and Decision Conference, 1996.

[7] C. Beck, R. D'Andrea, F. Paganini, W. M. Lu, and J. Doyle, "A state-space theory of uncertain systems," in IFAC Proceedings, 1996.

[8] A. J. L. P. Gahinet, A. Nemirovskii and M. Chilali, The LMI Control Toolbox, Beta-Release. The MathWorks Inc., 1994.

[9] F. D. L. El Ghaoui and R. Nikoukah, LMITOOL: A User-Friendly Interface for LMI Optimization. Not known at this time, 1995.

[10] M. Fliess, "Matrices de hankel," J. Math Pures et Appl., pp. 197-222, 1974.

[11] J. Willems, "Paradigms and puzzles in the theory of dynamical systems," IEEE Transactions on Automatic Control, pp. 259-294, 1991.

[12] S. Weiland, Theory of Approximation and Disturbance Attenuation for Linear Systems. PhD thesis, University of Groningen, 1991.

[13] C. Beck and J. C. Doyle, "Model reduction of behavioural systems," in Proc. IEEE Control and Decision Conference, 1993. 